\title{
Many-mode Floquet theoretical approach for coherent control of multiphoton dynamics driven by intense frequency-comb laser fields
}

\author{
Sang-Kil Son (손상길) $)^{1, *}$ and Shih-I Chu (朱時宜) $)^{1,2, \uparrow}$ \\ ${ }^{1}$ Department of Chemistry, University of Kansas, Lawrence, Kansas 66045, USA \\ ${ }^{2}$ Center for Theoretical Sciences, Department of Physics, National Taiwan University, \\ Taipei, Taiwan \\ (Received 22 February 2008; published 5 June 2008)
}

\begin{abstract}
We extend the many-mode Floquet theorem (MMFT) for the investigation of multiphoton resonance dynamics driven by intense frequency-comb laser fields. The frequency comb structure generated by a train of short laser pulses can be exactly represented by a combination of the main frequency and the repetition frequency. MMFT allows non-perturbative and exact treatment of the interaction of a quantum system with the frequency-comb laser fields. We observe simultaneous multiphoton resonance processes between a two-level system and frequency-comb laser. The multiphoton processes can be coherently controlled by tuning the laser parameters such as the carrier-envelope phase (CEP) shift. In particular, high-order harmonic generation shows immense enhancement by tuning the CEP shift, due to simultaneous multiphoton resonances.
\end{abstract}

DOI: 10.1103/PhysRevA.77.063406

PACS number(s): $33.80 . \mathrm{Rv}, 42.50 . \mathrm{Hz}, 42.65 . \mathrm{Ky}, 32.80 . \mathrm{Qk}$

\section{INTRODUCTION}

Recently, frequency-comb laser generated from the modelocked short-pulse laser has remarkably impacted on highprecision optical frequency measurement and synthesis [1-3], and enabled optical atomic clocks [4-6] and high sensitive molecular detection [7,8]. As a universal optical frequency-comb synthesizer, it provides the direct link between optical and microwave frequencies [9-11]. The femtosecond frequency-comb techniques are also stimulating new frontiers in ultrafast science $[12,13]$. Control of the waveform and phase evolution of few-cycle laser pulses $[10,14]$ provides a powerful new tool for the study of highly nonlinear optical phenomena that depend on the carrierenvelope phase (CEP), and the generation of soft-X-ray attosecond pulses by means of high-order harmonic generation (HHG) [13], etc.

More recently, the feasibility of the extension of the frequency comb structure and coherence into vacuumultraviolet (vuv) and/or extreme ultraviolet (xuv) regimes via HHG has attracted considerable attention $[15,16]$. It is speculated that the frequency comb structure may be retained in each of the higher harmonics. However, currently the experimental realization of the comb structure in higher harmonics has not yet been achieved due to some experimental resolution problems, with the exception of the third harmonics [16]. It is also not clear whether the frequency comb structure and coherence can survive in very high-order harmonics and in the situation where substantial ionization occurs in the presence of high intensity laser fields. To advance this field, we have recently presented $a b$ initio theoretical exploration of the frequency comb structure and coherence in the vuvxuv regimes via HHG [17]. The HHG spectrum driven by a sequence of laser pulses is calculated accurately and efficiently by propagating the time-dependent Schrödinger equa-

\footnotetext{
*sangkil@ku.edu

†sichu@ku.edu
}

tion by means of the time-dependent generalized pseudospectral method [18]. We explored the comb structure and coherence by varying the time interval between pulses, the number of pulses, and the laser intensity. We showed that each harmonic (from the first harmonic all the way to the cutoff) has a nested comb structure and this global pattern persists regardless of the time interval and the number of pulses, even in the presence of appreciable ionization.

In this paper, we present an $a b$ initio investigation of the coherent control of multiphoton resonance dynamics by means of intense frequency comb laser fields. In the limit of infinite number of pulses, we show that the many-mode Floquet theory (MMFT) [19-23] provides an exact formalism for nonperturbative treatment of the interaction of the frequency comb laser fields with atomic and molecular systems, allowing accurate treatment of the coherent control of multiphoton processes. We note that the original MMFT was developed for the exact treatment of multiphoton processes in the presence of quasiperiodic and/or polychromatic timedependent laser fields. In this paper, we extend the MMFT to the study of simultaneous multiphoton resonant excitations and coherent control of HHG enhancement driven by frequency comb laser fields.

The paper is organized as follows. In Sec. II, we present the MMFT for the treatment of the interaction of frequency comb laser fields with finite-level quantum systems. In Sec. III, we apply our theory to the study of the coherent control of multiphoton resonant dynamics of two-level systems by means of frequency comb laser fields. This is followed by the conclusion in Sec. IV.

\section{MMFT TREATMENT OF MULTIPHOTON EXCITATION BY MEANS OF FREQUENCY-COMB LASER}

Frequency-comb laser consists of spectral comb lines in the frequency domain $[24,25]$,

$$
\omega_{m}=m \omega_{r}+\omega_{\delta},
$$

where $\omega_{r}$ is the repetition angular frequency, $m$ is an integer index, and $\omega_{\delta}$ is the offset angular frequency $\left(0 \leq \omega_{\delta}<\omega_{r}\right)$. 
This frequency-comb laser can be generated by a temporal train of short laser pulses which have the carrier (fundamental) angular frequency $\omega_{c}$ and the time interval $\tau$ between pulses. Because the time period $\left(=2 \pi / \omega_{c}\right)$ of the carrier wave and the time duration $\tau\left(=2 \pi / \omega_{r}\right)$ of the pulse envelope are often incommensurate, there is the carrier-envelope phase (CEP) after each pulse: $\phi=\omega_{c} \tau$. Current mode-locking laser technique allows us to precisely control the CEP by including the pulse-to-pulse CEP shift $\Delta \phi$ that plays a key role in stabilizing of the comb structure [9]. The CEP shift $\Delta \phi$ determines all absolute positions of frequencies in the comb structure by setting the offset frequency $\omega_{\delta}=\Delta \phi / \tau$.

The electric field for a train of pulses with the CEP shift in the time domain [24] is expressed by

$$
E(t)=\sum_{n} f(t-n \tau) e^{i\left(\omega_{c} t-n \omega_{c} \tau+n \Delta \phi\right)}
$$

where $f(t)$ is the envelope function for one pulse in the time domain. In the ideal case that an infinite number of pulses are added up, Eq. (2) reduces to the summation of exponentials of discrete comb frequencies with their individual field amplitudes [5],

$$
E(t)=\sum_{m} E_{m} e^{i \omega_{m} t}
$$

where $E_{m}$ is the field amplitude corresponding to $\omega_{m}$ given by Eq. (1). See the Appendix for detailed derivation. Without loss of generality, we can rewrite these comb frequencies $\left\{\omega_{m}\right\}$ as

$$
\omega_{k}=\omega_{0}+k \omega_{r}
$$

where $k$ is an integer index and $\omega_{0}$ is the main angular frequency defined to be the frequency in $\left\{\omega_{m}\right\}$ whose field amplitude is the maximum in the frequency domain. In other words, $\omega_{0}$ is the closest frequency to $\omega_{c}$ among all the comb frequencies. The relation between $\omega_{0}$ and $\omega_{c}$ is given by

$$
\omega_{0}=\left[\frac{\omega_{c}-\omega_{\delta}}{\omega_{r}}\right] \omega_{r}+\omega_{\delta},
$$

where [ ] is the round function. Note that the fundamental frequency $\omega_{c}$ is not necessarily one of the comb frequencies nor does it equal $\omega_{0}$ in general. But if we choose $\omega_{c}=\omega_{0}$ or $\omega_{c} \in\left\{\omega_{k}\right\}$, then $\omega_{\delta}$ will be a reminder of $\omega_{c}$ divided by $\omega_{r}$.

If the envelope function $f(t)$ has the Gaussian form, then the infinite summation in Eq. (3) will reduce to the finite summation, and the field amplitudes can be explicitly determined. Let $f(t)=f_{0} e^{-t^{2} / 2 \sigma^{2}}$ where $f_{0}$ is the peak field amplitude at $t=0$ and $\sigma$ is the standard deviation of a Gaussian function. An integer number $N$ is chosen such that $E_{k} \approx 0$ when $|k|>N$. As a result, the electric field of $(2 N+1)$ comb frequencies is given by

$$
E(t)=\sum_{k=-N}^{N} E_{k} e^{i \omega_{k} t}
$$

where the field amplitude $E_{k}$ corresponding to $\omega_{k}$ is

$$
E_{k}=\frac{f_{0} \sigma \omega_{r}}{\sqrt{2 \pi}} e^{-\sigma^{2}\left(\omega_{0}-\omega_{c}+k \omega_{r}\right)^{2} / 2} .
$$

Detailed derivations are given in the Appendix.

To investigate the interaction of an atomic or molecular system with the comb laser generated by a finite number of pulses, Eq. (2) has been employed for solving the timedependent Schrödinger equation in the time domain [17]. On the other hand, for the ideal comb laser generated by an infinite number of pulses, Eq. (6) is more preferable to use, but it may require infinite duration time for the time propagation. Consequently, in order to consider the interaction with the ideal comb laser, it is more expedient to avoid the time propagation of wave functions.

The many-mode Floquet theory (MMFT) [19-23] can be applied to the solution of the polychromatic or quasiperiodic time-dependent Schrödinger equation without the time propagation of wave functions. Let us now consider the interaction of an atomic or molecular system with a linearly polarized frequency-comb laser $(\mathbf{E} \| \mathbf{z})$. The corresponding Hamiltonian including $(2 N+1)$ comb frequencies is given by,

$$
\begin{aligned}
H(\mathbf{r}, t) & =\hat{H}_{0}(\mathbf{r})-\sum_{k=-N}^{N} \mu(\mathbf{r}) \cdot \mathbf{E}_{k} \operatorname{Re}\left[e^{i \omega_{k} t}\right] \\
& =\hat{H}_{0}(\mathbf{r})-\sum_{k=-N}^{N} \hat{z} E_{k} \cos \omega_{k} t \\
& =\hat{H}_{0}(\mathbf{r})-\sum_{k=-N}^{N} \frac{1}{2} \hat{z} E_{k}\left[e^{i\left(\omega_{0}+k \omega_{r}\right) t}+e^{-i\left(\omega_{0}+k \omega_{r}\right) t}\right],
\end{aligned}
$$

where $\hat{H}_{0}(\mathbf{r})$ is the unperturbed Hamiltonian of the system and $\mu(\mathbf{r})$ is the electric dipole moment operator.

The MMFT states that any polychromatic (multi-color laser-field) problem has the exact solution in terms of the generalized many-mode quasienergy eigenvalues and eigenfunctions $[19,20]$. Because all the comb frequencies can be represented by two variables, $\omega_{0}$ and $\omega_{r}$, we can employ the two-mode Floquet theory with double Fourier components,

$$
|\alpha n m\rangle=|\alpha\rangle \otimes|n\rangle \otimes|m\rangle,
$$

where $\alpha$ is the system index, and $n$ and $m$ are Fourier components of $\omega_{0}$ and $\omega_{r}$, respectively. Then, the Hamiltonian can be expanded with double Fourier components of $n$ and $m$,

$$
H(\mathbf{r}, t)=\sum_{n, m} H^{[n, m]}(\mathbf{r}) e^{-i\left(n \omega_{0}+m \omega_{r}\right) t},
$$

where 


$$
\begin{gathered}
H^{[0,0]}=\hat{H}_{0}, \\
H^{[-1,0]}=H^{[+1,0]}=-\frac{1}{2} \hat{z} E_{0}, \\
H^{[-1,-1]}=H^{[+1,+1]}=-\frac{1}{2} \hat{z} E_{1}, \quad H^{[-1,+1]}=H^{[+1,-1]}=-\frac{1}{2} \hat{z} E_{-1}, \\
H^{[-1,-2]}=H^{[+1,+2]}=-\frac{1}{2} \hat{z} E_{2}, \quad H^{[-1,+2]}=H^{[+1,-2]}=-\frac{1}{2} \hat{z} E_{-2}, \\
\vdots \vdots \\
H^{[-1,-N]}=H^{[+1,+N]}=-\frac{1}{2} \hat{z} E_{N}, \quad H^{[-1,+N]}=H^{[+1,-N]}=-\frac{1}{2} \hat{z} E_{-N} .
\end{gathered}
$$

The MMFT allows the exact transformation of the timedependent problem into an equivalent time-independent infinite-dimensional generalized Floquet matrix eigenvalue problem,

$$
\sum_{\beta} \sum_{n^{\prime}} \sum_{m^{\prime}}\left\langle\alpha n m\left|H_{F}\right| \beta n^{\prime} m^{\prime}\right\rangle\left\langle\beta n^{\prime} m^{\prime} \mid \lambda\right\rangle=\lambda\langle\alpha n m \mid \lambda\rangle,
$$

where $\lambda$ is the quasienergy eigenvalue and $|\lambda\rangle$ is the corresponding eigenvector. The two-mode Floquet matrix $H_{F}$ is constructed by

$$
\left\langle\alpha n m\left|H_{F}\right| \beta n^{\prime} m^{\prime}\right\rangle=H_{\alpha \beta}^{\left[n-n^{\prime}, m-m^{\prime}\right]}+\left(n \omega_{0}+m \omega_{r}\right) \delta_{\alpha, \beta} \delta_{n, n^{\prime}} \delta_{m, m^{\prime}},
$$

where

$$
\begin{aligned}
& H_{\alpha \beta}^{\left[n-n^{\prime}, m-m^{\prime}\right]}=\left\langle\alpha\left|H^{\left[n-n^{\prime}, m-m^{\prime}\right]}\right| \beta\right\rangle \\
&= \varepsilon_{\alpha} \delta_{\alpha, \beta} \delta_{n, n^{\prime}} \delta_{m, m^{\prime}} \\
&+\sum_{k=-N}^{N} V_{\alpha \beta}^{(k)}\left(\delta_{n+1, n^{\prime}} \delta_{m+k, m^{\prime}}+\delta_{n-1, n^{\prime}} \delta_{m-k, m^{\prime}}\right) \\
& \varepsilon_{\alpha}=\left\langle\alpha\left|\hat{H}_{0}\right| \alpha\right\rangle \quad \text { and } \quad V_{\alpha \beta}^{(k)}=-\frac{1}{2} E_{k}\langle\alpha|\hat{z}| \beta\rangle .
\end{aligned}
$$

The $H_{F}$ matrix has a block band-diagonal structure with infinite-dimensional diagonal blocks and subdiagonal blocks corresponding to interactions between the quantum system and the $(2 N+1)$ comb frequencies. The structure of $H_{F}$ for the linearly polarized case is as follows:

$$
H_{F}=\left(\begin{array}{ccccccc}
\ddots & & & & & \vdots & \\
& A+2 \omega_{r} I & B_{1} & B_{2} & B_{3} & B_{4} & \ldots \\
& B_{1}^{T} & A+\omega_{T} I & B_{1} & B_{2} & B_{3} & \\
& B_{2}^{T} & B_{1}^{T} & A & B_{1} & B_{2} & \\
& B_{3}^{T} & B_{2}^{T} & B_{1}^{T} & A-\omega_{r} I & B_{1} & \\
\cdots & B_{4}^{T} & B_{3}^{T} & B_{2}^{T} & B_{1}^{T} & A-2 \omega_{r} I & \\
& \vdots & & & & & \ddots
\end{array}\right),
$$

where $A$ is a block tridiagonal matrix and $B_{k}$ is a block offdiagonal matrix,

$$
A=\left(\begin{array}{ccccccc}
\ddots & & & & & \vdots & \\
& Z+2 \omega_{0} I & X & 0 & 0 & 0 & \cdots \\
& X & Z+\omega_{0} I & X & 0 & 0 & \\
& 0 & X & Z & X & 0 & \\
& 0 & 0 & X & Z-\omega_{0} I & X & \\
\cdots & 0 & 0 & 0 & X & Z-2 \omega_{0} I & \\
& \vdots & & & & & \ddots
\end{array}\right)
$$

The matrices of $X, Y_{k}$, and $Z$ which form blocks in $A$ and $B$ have the following forms: 

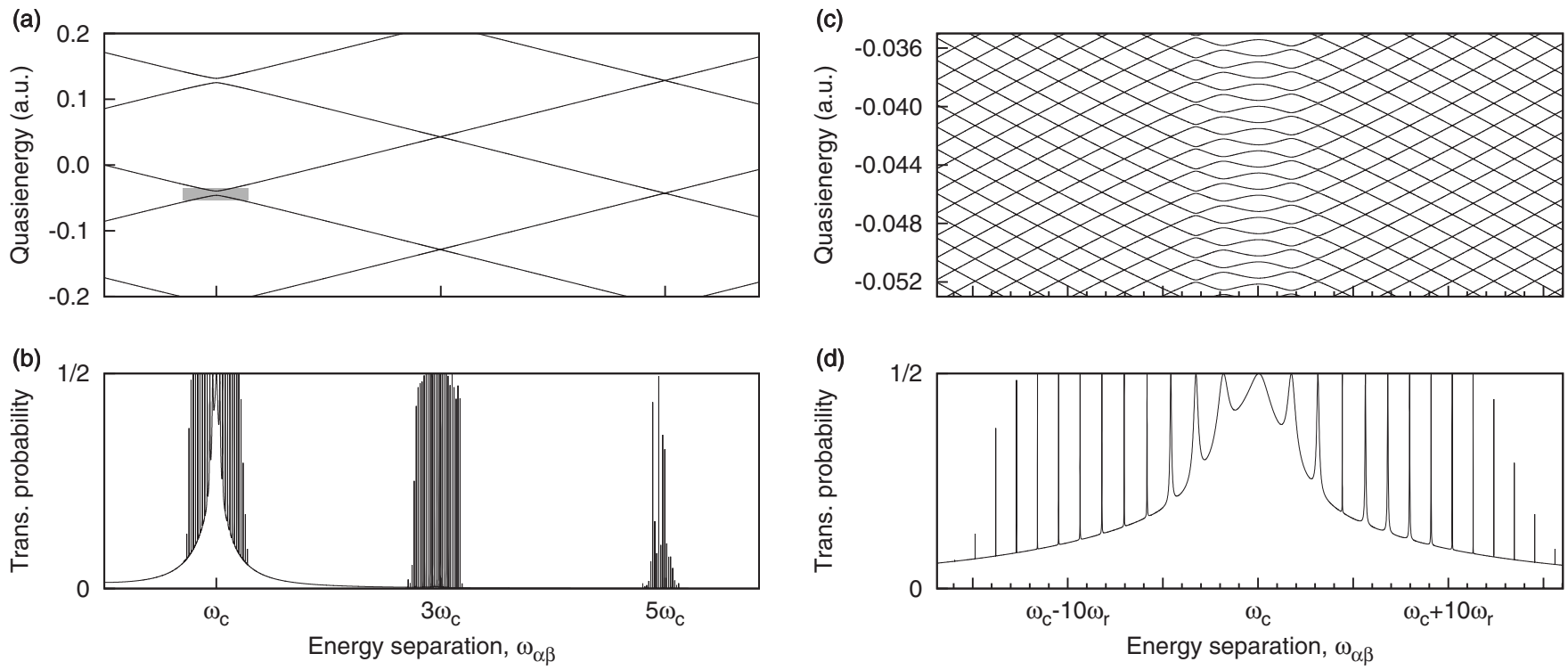

FIG. 1. (a) Quasienergies and (b) time-averaged transition probabilities as a function of energy separation, for the two-level system driven by frequency-comb laser fields with peak intensity $2.5 \times 10^{15} \mathrm{~W} / \mathrm{cm}^{2}$, carrier frequency $563.5 \mathrm{THz}$, and repetition frequency $10 \mathrm{THz}$ of 20 fs FWHM Gaussian pulses. (c) Enlarged quasienergy plots in the one-photon resonance regime corresponding to the gray box in (a). (d) The corresponding time-averaged transition probabilities.

$$
\begin{gathered}
X=\left(\begin{array}{cccc}
0 & V_{\alpha \beta}^{(0)} & V_{\alpha \gamma}^{(0)} & \cdots \\
V_{\beta \alpha}^{(0)} & 0 & V_{\beta \gamma}^{(0)} & \\
V_{\gamma \alpha}^{(0)} & V_{\gamma \beta}^{(0)} & 0 & \\
\vdots & & & \ddots
\end{array}\right), Y_{k}=\left(\begin{array}{cccc}
0 & V_{\alpha \beta}^{(k)} & V_{\alpha \gamma}^{(k)} & \cdots \\
V_{\beta \alpha}^{(k)} & 0 & V_{\beta \gamma}^{(k)} & \\
V_{\gamma \alpha}^{(k)} & V_{\gamma \beta}^{(k)} & 0 & \\
\vdots & & & \ddots
\end{array}\right) \\
Z=\left(\begin{array}{cccc}
\varepsilon_{\alpha} & 0 & 0 & \cdots \\
0 & \varepsilon_{\beta} & 0 & \\
0 & 0 & \varepsilon_{\gamma} & \\
\vdots & & & \ddots
\end{array}\right)
\end{gathered}
$$

After solving the eigenvalue problem of the generalized Floquet matrix, the time-averaged transition probability can be computed from the quasienergy eigenvectors [20],

$$
\bar{P}_{\alpha \rightarrow \beta}=\sum_{n, m} \sum_{\gamma, n^{\prime}, m^{\prime}}\left|\left\langle\beta n m \mid \lambda_{\gamma n^{\prime} m^{\prime}}\right\rangle\left\langle\lambda_{\gamma n^{\prime} m^{\prime}} \mid \alpha 00\right\rangle\right|^{2},
$$

which has the maximum value of $\frac{1}{2}$ at avoided crossings of quasienergies associated with multiphoton resonance transitions [21]. The induced dipole moment can be likewise expanded in double Fourier series,

$$
\mathbf{d}(t)=\sum_{n, m} \mathbf{d}_{n, m} e^{-i\left(n \omega_{0}+m \omega_{r}\right) t}
$$

If $n$ and $m$ are given, the angular frequency $\omega$ is determined by $\omega=n \omega_{0}+m \omega_{r}$. The HHG power spectra in the length form can be expressed by [26]

$$
P\left(n \omega_{0}+m \omega_{r}\right)=\left|\mathbf{d}_{n, m}\right|^{2}=\left|\sum_{n^{\prime}, m^{\prime}}\left\langle\lambda_{\beta, n^{\prime}-n, m^{\prime}-m}|\hat{z}| \lambda_{\alpha, n^{\prime}, m^{\prime}}\right\rangle\right|^{2} .
$$

Here harmonic order is defined by $\omega / \omega_{c}$ and can be a fractional number because of the comb structure of frequencies.

\section{NUMERICAL RESULTS AND DISCUSSIONS}

In this section, we present a case study of the multiphoton resonance enhancement of a two-level system driven by frequency-comb laser fields. The laser parameters used are peak intensity $2.5 \times 10^{15} \mathrm{~W} / \mathrm{cm}^{2}$, carrier frequency 563.5 $\mathrm{THz}$ (corresponding to $\omega_{c}=0.0856454$ a.u., and wavelength $532 \mathrm{~nm}$ ), and repetition frequency $10 \mathrm{THz}$ (corresponding to $\omega_{r}=1.51983 \times 10^{-3}$ a.u. and pulse separation $\tau=0.1 \mathrm{ps})$ generated from a train of Gaussian pulses with 20 fs full width at half maximum (FWHM). The main frequency $\omega_{0}$ is set to be the carrier frequency $\omega_{c}$, so that the offset angular frequency $\omega_{\delta}$ is chosen to be a reminder of $\omega_{c}$ divided by $\omega_{r}$. For the two-level system, the transition dipole moment $\langle\alpha|\hat{z}| \beta\rangle=0.1$ a.u. is used. Figure 1(a) shows the two-mode quasienergies, and Fig. 1(b) shows the timeaveraged transition probabilities as a function of energy separation of the two-level system, $\hbar \omega_{\alpha \beta}=\varepsilon_{\beta}-\varepsilon_{\alpha}$, varying from 0.0 to 0.5 a.u. Multiphoton resonances are found near odd numbers of $\omega_{c}$ similar to the one-mode Floquet calculation [27]. However, a cluster of resonances are observed in the vicinity of resonance positions due to the comb structure of frequencies. The quasiperiodic structure of the quasienergies can be represented by $[20,21]$

$$
\lambda_{\gamma n m}=\lambda_{\gamma}+n \omega_{0}+m \omega_{r},
$$

where $n$ is an even integer when $\gamma=\alpha$ and an odd integer when $\gamma=\beta$ due to parity consideration. Because of multiples of $\omega_{r}$, the quasienergy plot becomes more complicated with avoided crossings as shown in Fig. 1(c), where the onephoton dominant resonance pattern is enlarged from the gray box in Fig. 1(a). The corresponding transition probabilities are shown in Fig. 1(d).

Many features of the frequency-comb structure can be explained by simultaneous multiphoton resonances that oc- 
cur due to the multitude of frequencies at the same time. Let us consider an $n$-photon resonance condition of a monochromatic frequency $\omega$,

$$
\omega_{\mathrm{res}}=n \omega,
$$

where $\omega_{\text {res }}$ is the resonance frequency. On the other side, when one comb frequency $\omega$ is on $n$-photon resonance, many combinations of comb frequencies can simultaneously contribute to that multiphoton resonance. For example, if $\omega_{\text {res }}=n \omega$ where $\omega \in\left\{m \omega_{r}+\omega_{\delta}\right\}$, then a combination of $(n-2) \omega, \omega-\omega_{r}$, and $\omega+\omega_{r}$ is also on resonance with $\omega_{\text {res }}$. Thus all combinations of $\omega$ with $\pm l \omega_{r}(l$ :integer $)$ are also contributed to the resonance. Consequently, the $n$-photon resonance condition with frequency combs can be expressed by the congruence relation of real numbers,

$$
\omega_{\text {res }} \equiv n \omega\left(\bmod \omega_{r}\right) \text {. }
$$

Equation (24) means the reminders of $\omega_{\text {res }}$ and $n \omega$ are the same when divided by $\omega_{r}$.

Now, we explore the optimization of the multiphoton resonance processes by tuning the CEP shift $\Delta \phi$. We note that changing the CEP shift, the resonance processes can be easily achieved because $\Delta \phi$ determines absolute comb positions more precisely than $\omega_{c}$ and even $\omega_{r}\left(\omega_{\delta}=\omega_{r} \Delta \phi / 2 \pi\right)$. For example, with the laser parameters used in Fig. 1, 1\% variation on $\Delta \phi$ yields $0.018 \%$ changes on frequencies, which emphasizes that the control of the CEP shift in comb laser is much easier than the control of the carrier frequency in one-mode (monochromatic) laser to reach the resonance frequency. Also the resonance position will be retained when the phases vary because the pulse energy is conserved. Moreover, when the CEP shift is varied, it is possible to achieve $n$ times of $n$-photon resonance, where $n$ is an odd number. If comb frequencies with the offset $\omega_{\delta}$ are $n$-photon resonant with $\omega_{\text {res }}$,

$$
\begin{aligned}
\omega_{\mathrm{res}} & \equiv n\left(m \omega_{r}+\omega_{\delta}\right) \quad\left(\bmod \omega_{r}\right) \\
& \equiv n \omega_{\delta} \quad\left(\bmod \omega_{r}\right),
\end{aligned}
$$

then $\omega_{\delta}+\frac{j}{n} \omega_{r}(j$ : integer, $0 \leq j<n)$ are also $n$-photon resonant with $\omega_{\text {res }}$, leading to simultaneous multiphoton resonances, because

$$
n\left(m \omega_{r}+\omega_{\delta}+\frac{j}{n} \omega_{r}\right) \equiv n \omega_{\delta} \quad\left(\bmod \omega_{r}\right) .
$$

Equations (25) and (26) show that comb frequencies with $\omega_{\delta}$ and with $\omega_{\delta}+\frac{j}{n} \omega_{r}$ have the same reminder as $\omega_{\text {res }}$ when divided by $\omega_{r}$. The CEP shifts of comb frequencies with the offset $\omega_{\delta}+\frac{j}{n} \omega_{r}$ are given by

$$
\Delta \phi_{j}=2 \pi\left(\frac{\omega_{\delta}}{\omega_{r}}+\frac{j}{n}\right) \quad(0 \leq j<n),
$$

so that resonance peak positions of $\Delta \phi_{j}$ are separated by $2 \pi / n$. Figure 2(a) shows the quasienergies and Fig. 2(b) shows the time-averaged transition probabilities as a function of the CEP shift. The energy separation is fixed at $\omega_{\alpha \beta}=0.25$ a.u. which corresponds to the three-photon dominant resonance regime $\left(3 \omega_{c} \approx \omega_{\alpha \beta}\right)$, and $\Delta \phi$ is varied

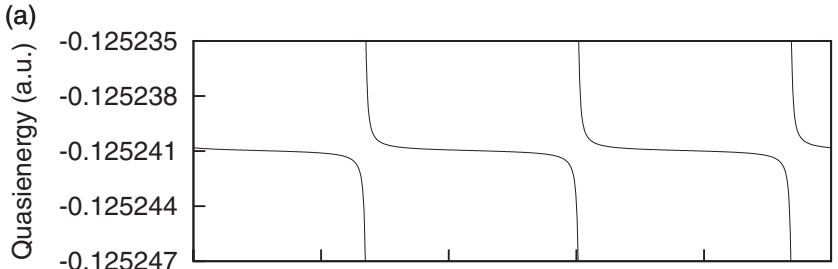

(b)

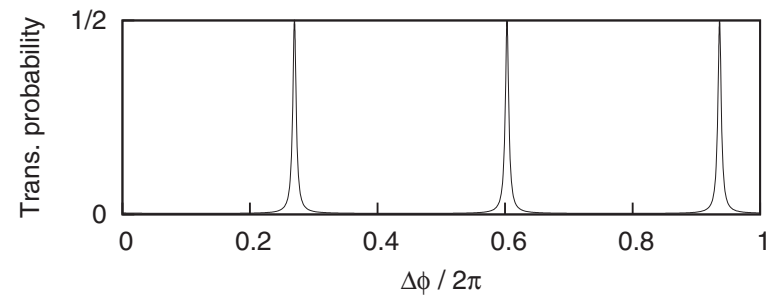

FIG. 2. (a) Plots of quasienergies and (b) time-averaged transition probabilities as a function of the CEP shift $\Delta \phi$. It shows three resonance positions separated by $2 \pi / 3$ due to three-photon dominant resonance.

$(0 \leq \Delta \phi<2 \pi)$. Other laser parameters are the same as those in Fig. 1. Shown in Fig. 2 is the case of three-photon dominant resonance. Here the resonance can be achieved three times by varying the CEP shift, and these three peaks are separated by $2 \pi / 3$ exactly.

In Fig. 3, we present the HHG power spectrum of twolevel systems driven by frequency-comb laser. The laser parameters are the same as those in Fig. 1 and the energy separation is fixed at $\omega_{\alpha \beta}=0.25$ a.u. For comparison, HHG driven by one-mode laser with the same carrier frequency and the corresponding root-mean-square field strength is plotted with the dots in Fig. 3, computed by one-mode Floquet calculation. Note that HHG spectrum driven by comb laser is orders of magnitude higher than that by one-mode laser. In addition, HHG driven by comb laser forms a nested comb structure within each of the harmonics, due to the constructive interferences among the sequence of induced di-

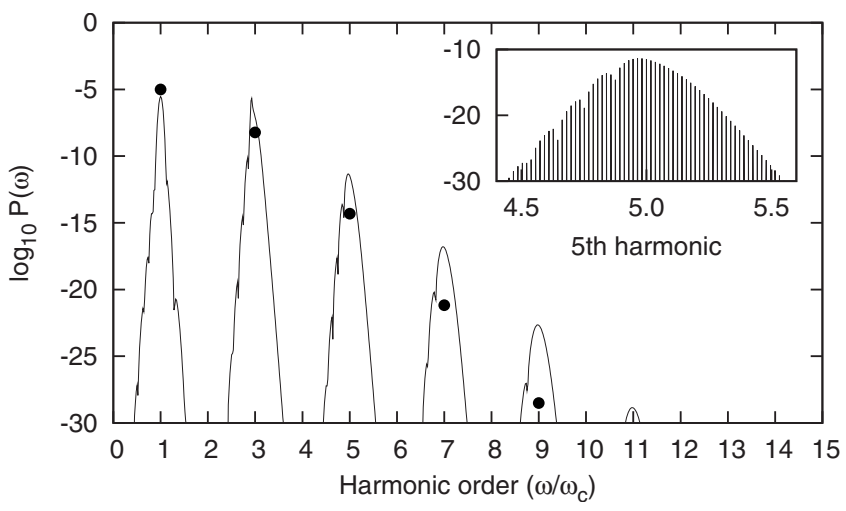

FIG. 3. HHG power spectrum of two-level systems driven by frequency-comb laser. Each harmonic has a nested comb structure as clearly shown in the inset for the fifth harmonic. For clarity, all comb peaks are connected by a line. The laser parameters used are peak intensity $2.5 \times 10^{15} \mathrm{~W} / \mathrm{cm}^{2}$, carrier frequency $563.5 \mathrm{THz}$, and repetition frequency $10 \mathrm{THz}$ of $20 \mathrm{fs}$ FWHM Gaussian pulses. Dots represent HHG spectra driven by the corresponding one-mode laser. 


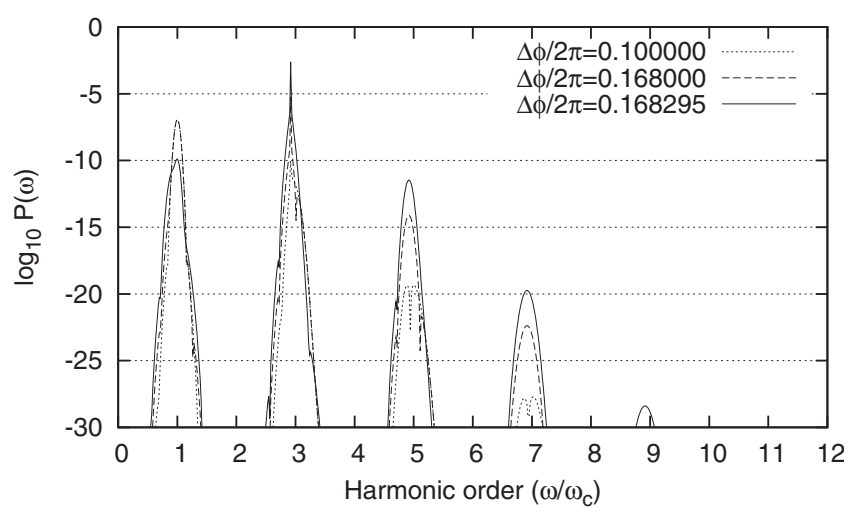

FIG. 4. Enhancement of HHG by varying the CEP shift with peak intensity $1 \times 10^{14} \mathrm{~W} / \mathrm{cm}^{2}$. For clarity, HHG peaks of the comb structure are connected by a line.

poles generated by the incident sequence of laser pulses [17]. For instance, the comb structure within the fifth-order harmonic is shown in the inset in Fig. 3. Each of the harmonic orders has the same repetition angular frequency $\omega_{r}$ and the offset angular frequency $n \omega_{\delta}$ for the $n$ th-order harmonic. The frequency comb spectrum for the $n$ th-order harmonic can be expressed with $\omega_{0}$,

$$
\omega_{k}^{\prime}=n \omega_{0}+k \omega_{r},
$$

or expressed with $\omega_{\delta}$,

$$
\omega_{m}^{\prime}=n \omega_{\delta}+m \omega_{r}
$$

where $m$ and $k$ are an integer index.

Finally, we investigate the coherent control and enhancement of HHG power spectra by tuning the CEP shift to achieve multiphoton resonances. Figure 4 shows the enhancement of HHG by varying the CEP shift, $\Delta \phi / 2 \pi=0.1$ (off-resonance), 0.168 , and 0.168295 (near-resonance) with peak intensity $1 \times 10^{14} \mathrm{~W} / \mathrm{cm}^{2}$. Other parameters for comb laser and the system are the same as those in Fig. 3. Table I lists the power spectrum values of the maximum peak for the near- and off-resonance cases with several peak intensities. Figure 4 and Table I show that HHG peaks can be dramati- cally enhanced by varying the CEP shift due to simultaneous multiphoton resonances. In the case of $n$-photon resonance processes, the system can easily reach the excited state by the absorption of $n$ photons and the higher-order $(>n)$ harmonic spectra can be also enhanced. The power spectra of $I=1 \times 10^{14} \mathrm{~W} / \mathrm{cm}^{2}$ are enhanced by about $10^{8}$ times. For the case of $I=1 \times 10^{15} \mathrm{~W} / \mathrm{cm}^{2}$, an enhancement factor is about $10^{5}$ times, while for the case of $I=2.5 \times 10^{15} \mathrm{~W} / \mathrm{cm}^{2}$, it is about $10^{3}$ times. Note that similar enhancement is observed at other resonance positions of the CEP shift. For example, Fig. 2(b) indicates three different resonance positions of $\Delta \phi$ at $I=2.5 \times 10^{15} \mathrm{~W} / \mathrm{cm}^{2}$. The enhancement factors of the HHG power spectrum with $\Delta \phi / 2 \pi=0.2697$, or $0.2697+1 / 3$, or $0.2697+2 / 3$ are all the same about $10^{3}$ times.

\section{CONCLUSION}

In this paper, we show that a train of an infinite number of short laser pulses generates spectral combs in the frequency domain, that can be exactly expressed in terms of the main frequency and the repetition frequency. The many-mode Floquet theory utilizing those two frequencies is extended to accurately solve the interaction between the quantum system and frequency-comb laser. It is shown that there are simultaneous multiphoton resonance processes between the system and comb laser, and that they can be optimally controlled by tuning the pulse-to-pulse CEP shift. HHG driven by intense frequency-comb laser has the comb structure with the same repetition frequency and different offset for each harmonic. Moreover, HHG can be dramatically enhanced by tuning the CEP shift due to simultaneous multiphoton resonance among all the comb frequencies. Extension of the present work to the study of the coherent control of atomic and molecular processes is in progress.

\section{ACKNOWLEDGMENTS}

This work was partially supported by the Chemical Sciences, Geosciences and Biosciences Division of the Office of Basic Energy Sciences, Office of Sciences, U.S. Department

TABLE I. Effects on the power spectra by varying the CEP shift $\Delta \phi . q$ is the harmonic order of the maximum peak for each harmonic and $P\left(q \omega_{c}\right)$ is the corresponding to power spectrum at $\omega=q \omega_{c}$. The label A indicates $\Delta \phi / 2 \pi=0.1$ that shows off-resonance cases, while B indicates the near-resonance cases: $\Delta \phi / 2 \pi=0.168295,0.20653$, and 0.2697 for $1 \times 10^{14}, 1 \times 10^{15}$, and $2.5 \times 10^{15} \mathrm{~W} / \mathrm{cm}^{2}$, respectively.

\begin{tabular}{|c|c|c|c|c|c|c|c|c|c|c|c|}
\hline \multicolumn{4}{|c|}{$1 \times 10^{14} \mathrm{~W} / \mathrm{cm}^{2}$} & \multicolumn{4}{|c|}{$1 \times 10^{15} \mathrm{~W} / \mathrm{cm}^{2}$} & \multicolumn{4}{|c|}{$2.5 \times 10^{15} \mathrm{~W} / \mathrm{cm}^{2}$} \\
\hline \multicolumn{2}{|r|}{ A } & \multicolumn{2}{|r|}{ B } & \multicolumn{2}{|c|}{ A } & \multicolumn{2}{|r|}{ B } & \multicolumn{2}{|c|}{ A } & \multicolumn{2}{|r|}{ B } \\
\hline$q$ & $P\left(q \omega_{c}\right)$ & $q$ & $P\left(q \omega_{c}\right)$ & $q$ & $P\left(q \omega_{c}\right)$ & $q$ & $P\left(q \omega_{c}\right)$ & $q$ & $P\left(q \omega_{c}\right)$ & $q$ & $P\left(q \omega_{c}\right)$ \\
\hline 2.92 & $9.15[-11]$ & 2.92 & $2.50[-3]$ & 2.92 & $7.07[-8]$ & 2.92 & $2.41[-3]$ & 2.92 & $1.33[-6]$ & 2.92 & $2.01[-3]$ \\
\hline 5.00 & $4.53[-20]$ & 4.91 & $3.42[-12]$ & 5.00 & $6.62[-15]$ & 4.93 & $3.39[-10]$ & 5.00 & $1.28[-12]$ & 4.94 & $2.07[-9]$ \\
\hline 7.02 & $1.99[-28]$ & 6.92 & $1.83[-20]$ & 7.00 & $3.10[-21]$ & 6.93 & $1.82[-16]$ & 7.00 & $4.13[-18]$ & 6.95 & $6.82[-15]$ \\
\hline \multirow[t]{3}{*}{9.03} & $5.58[-37]$ & 8.92 & $4.07[-29]$ & 9.01 & $6.00[-28]$ & 8.92 & $3.99[-23]$ & 9.01 & $5.30[-24]$ & 8.95 & $9.40[-21]$ \\
\hline & & & & 11.02 & $5.45[-35]$ & 10.92 & $4.04[-30]$ & 11.00 & $3.14[-30]$ & 10.95 & $5.95[-27]$ \\
\hline & & & & & & & & 13.03 & $1.05[-36]$ & 12.95 & $1.96[-33]$ \\
\hline
\end{tabular}
The number in brackets indicates the power of 10 . 
of Energy, by U.S. National Science Foundation, by National Taiwan University, and by National Science Council (NSC 97-2112-M-002-003-MY3), Taiwan. S. -K. S. would like to acknowledge also CRDF Grant No. UZP1-2817-TA-06 for the partial support of this work.

\section{APPENDIX: FIELD EXPRESSION FOR FREQUENCY- COMB LASER}

The electric field for a train of pulses with the CEP shift $\Delta \phi$ in the time domain [Eq. (2)] is expressed by

$$
E(t)=\sum_{n=1}^{N_{p}} f(t-n \tau) e^{i\left(\omega_{c} t-n \omega_{c} \tau+n \Delta \phi\right)},
$$

where $N_{p}$ is the number of pulses and $f(t)$ is the envelope for one pulse in the time domain. After the Fourier transform, the electric field in the frequency domain is given by [24]

$$
\widetilde{E}(\omega)=\widetilde{f}\left(\omega-\omega_{c}\right) \sum_{n=1}^{N_{p}} e^{-i n(\omega \tau-\Delta \phi)},
$$

where $\tilde{f}(\omega)=\int f(t) e^{-i \omega t} d t$ determines the field strength in the spectrum as the envelope function in the frequency domain. The summation of the exponentials generates frequency comb structure because they are added up with constructive interferences only at $\omega \tau-\Delta \phi=2 \pi m$ with an arbitrary integer $m$ [9]. In other words, the summation becomes a series of frequency lines at $\omega=\omega_{m}$ such that

$$
\omega_{m}=\frac{2 \pi m}{\tau}+\frac{\Delta \phi}{\tau}=m \omega_{r}+\omega_{\delta}
$$

which is shown as the comb frequency expression in Eq. (1). We can rewrite these comb frequencies with the main angular frequency $\omega_{0}$ [Eq. (4)],

$$
\omega_{k}=\omega_{0}+k \omega_{r}
$$

because it is more convenient to derive the field expression of Eq. (6) with respect to $\omega_{0}$.

When the number of pulses increases, the spectral width of each frequency comb becomes narrower due to quantum interference $[3,17]$. In the ideal case that an infinite number of pulses are added up, the summation part yields a series of delta functions centered at each $\omega_{k}$,

$$
\lim _{N_{p} \rightarrow \infty} \sum_{n=1}^{N_{p}} e^{-i n(\omega \tau-\Delta \phi)}=\frac{2 \pi}{\tau} \sum_{k=-\infty}^{\infty} \delta\left(\omega-\omega_{k}\right) .
$$

Thus the ideal comb laser field in the frequency domain can be represented by

$$
\widetilde{E}^{\mathrm{o}}(\omega)=\widetilde{f}\left(\omega-\omega_{c}\right) \omega_{r} \sum_{k=-\infty}^{\infty} \delta\left(\omega-\omega_{k}\right) .
$$

After applying the inverse Fourier transform to $\widetilde{E}^{\mathrm{o}}(\omega)$, we obtain a time-domain expression for the electric field of the ideal comb laser,

$$
E^{\mathrm{o}}(t)=\frac{\omega_{r}}{2 \pi} \sum_{k=-\infty}^{\infty} \int_{-\infty}^{\infty} \delta\left(\omega-\omega_{k}\right) \tilde{f}\left(\omega-\omega_{c}\right) e^{i \omega t} d \omega=\sum_{k=-\infty}^{\infty} E_{k} e^{i \omega_{k} t},
$$

where $E_{k}=\tilde{f}\left(\omega_{k}-\omega_{c}\right) \omega_{r} / 2 \pi$ represents the field amplitude of $\omega_{k}$. This summation of exponentials of the angular frequencies $\left\{\omega_{k}\right\}$ with their field amplitudes $\left\{E_{k}\right\}$ is coherently added up and then generates the ideal comb structure in the frequency domain. Inversely, this expression plots an infinite number of train pulses in the time domain, i.e., Eq. (A7) is identical to Eq. (A1) if $N_{p}$ goes to infinity.

With the help of the envelope function such as a Gaussian function, it is possible to truncate Eq. (A7) to have a finite summation. In this case, $N$ is chosen such that $E_{k} \approx 0$ when $|k|>N$, and then the electric field expression of the comb laser is reduced to the summation of a finite set of $(2 N+1)$ angular frequencies shown in Eq. (6),

$$
E^{\mathrm{o}}(t)=\sum_{k=-N}^{N} E_{k} e^{i \omega_{k} t}
$$

If a Gaussian function is used for the envelope, $f(t)=f_{0} e^{-t^{2} / 2 \sigma^{2}}$, the field amplitude $E_{k}$ in Eq. (7) can be derived explicitly as

$$
\begin{aligned}
E_{k} & =\frac{\omega_{r}}{2 \pi} \int_{-\infty}^{\infty} f_{0} e^{-t^{\prime 2} / 2 \sigma^{2}} e^{-i\left(\omega_{k}-\omega_{c}\right) t^{\prime}} d t^{\prime} \\
& =\frac{f_{0} \sigma \omega_{r}}{\sqrt{2 \pi}} e^{-\sigma^{2}\left(\omega_{0}-\omega_{c}+k \omega_{r}\right)^{2} / 2} .
\end{aligned}
$$

Note that the standard deviation $\sigma$ of a Gaussian function is related to $\mathrm{FWHM}=2 \sigma \sqrt{2 \ln 2}$.
[1] M. Fischer et al., Phys. Rev. Lett. 92, 230802 (2004).

[2] H. S. Margolis, G. P. Barwood, G. Huang, H. A. Klein, S. N. Lea, K. Szymaniec, and P. Gill, Science 306, 1355 (2004).

[3] S. Witte, R. T. Zinkstok, W. Ubachs, W. Hogervorst, and K. S. E. Eikema, Science 307, 400 (2005).

[4] M. Takamoto, F.-L. Hong, R. Higashi, and H. Katori, Nature (London) 435, 321 (2005).

[5] T. Udem, R. Holzwarth, and T. W. Hänsch, Nature (London) 416, 233 (2002).
[6] S. A. Diddams et al., Science 293, 825 (2001).

[7] M. J. Thorpe, K. D. Moll, R. J. Jones, B. Safdi, and J. Ye, Science 311, 1595 (2006).

[8] S. A. Diddams, L. Hollberg, and V. Mbele, Nature (London) 445, 627 (2007).

[9] D. J. Jones, S. A. Diddams, J. K. Ranka, A. Stentz, R. S. Windeler, J. L. Hall, and S. T. Cundiff, Science 288, 635 (2000).

[10] M. Niering et al., Phys. Rev. Lett. 84, 5496 (2000). 
[11] R. Holzwarth, T. Udem, T. W. Hänsch, J. C. Knight, W. J. Wadsworth, and P. S. J. Russell, Phys. Rev. Lett. 85, 2264 (2000).

[12] M. Hentschel, R. Kienberger, C. Spielmann, G. A. Reider, N. Milosevic, T. Brabec, P. Corkum, U. Heinzmann, M. Drescher, and F. Krausz, Nature (London) 414, 509 (2001).

[13] M. Drescher, M. Hentschel, R. Kienberger, G. Tempea, C. Spielmann, G. A. Reider, P. B. Corkum, and F. Krausz, Science 291, 1923 (2001).

[14] A. Apolonski, A. Poppe, G. Tempea, C. Spielmann, T. Udem, R. Holzwarth, T. W. Hänsch, and F. Krausz, Phys. Rev. Lett. 85, 740 (2000).

[15] C. Gohle, T. Udem, M. Herrmann, J. Rauschenberger, R. Holzwarth, H. A. Schuessler, F. Krausz, and T. W. Hänsch, Nature (London) 436, 234 (2005).

[16] R. J. Jones, K. D. Moll, M. J. Thorpe, and J. Ye, Phys. Rev.
Lett. 94, 193201 (2005).

[17] J. J. Carrera, S.-K. Son, and S. I. Chu, Phys. Rev. A 77, 031401(R) (2008).

[18] X. M. Tong and S. I. Chu, Chem. Phys. 217, 119 (1997).

[19] S. I. Chu and D. A. Telnov, Phys. Rep. 390, 1 (2004).

[20] T. S. Ho, S. I. Chu, and J. V. Tietz, Chem. Phys. Lett. 96, 464 (1983).

[21] T. S. Ho and S. I. Chu, J. Phys. B 17, 2101 (1984).

[22] T. S. Ho and S. I. Chu, Phys. Rev. A 31, 659 (1985).

[23] T. S. Ho and S. I. Chu, Phys. Rev. A 32, 377 (1985).

[24] S. T. Cundiff, J. Ye, and J. L. Hall, Rev. Sci. Instrum. 72, 3749 (2001).

[25] S. T. Cundiff and J. Ye, Rev. Mod. Phys. 75, 325 (2003).

[26] D. A. Telnov, J. Y. Wang, and S. I. Chu, Phys. Rev. A 52, 3988 (1995).

[27] J. H. Shirley, Phys. Rev. 138, B979 (1965). 